\title{
Does dimensionality distinguish difficult questions?
}

\author{
Evidence of Non-Mean-Field Low-Temperature Behavior in the Edwards-Anderson Spin-Glass Model
}

Burcu Yucesoy, Helmut G. Katzgraber, and Jon Machta,

Physical Review Letters 109, 177204 (2012).

\section{Recommended with a commentary by A. Alan Middleton, Syracuse University}

Many of the current challenges in science at the scales from nanometers through meters are posed by statistically uniform but heterogeneous assemblies. Constructing and testing a quantum computer with many qubits, studying the transport of information and mechanical forces in biological systems, and experimenting with mixtures of colloidal or granular particles all require characterization and modeling of non-uniform systems. Physicists might wish to find the universal features and scaling properties of large collections of such logic gates, cells, or particles. But such searches confront the fact that universal features are much more difficult to determine in the presence of disorder than, say, for a uniform Ising ferromagnet. It can be unclear how to find and coarse grain an order parameter. The example of quantum computing built on generic interactions is particularly complex, as deciding whether a quantum computer is faster than a classical computer requires considered comparisons of both the complexity of the device and of the purportedly difficult problem that it is solving.

Models of spin glass materials provide a well-studied and important guide to general questions about qualitative features and universality in heterogeneous systems. Beyond any interest in the materials that directly realize the spin glass model in bulk (or possibly on the surface of pure materials), these models are used as prototypes both for other disordered materials and as tests of optimization algorithms. As low-temperature spin glass models can be used to encode NP-complete problems and to explore glassy dynamics, it is difficult to use numerics to verify phenomenological pictures. Analytic progress has been very difficult. So even in this prototypical model, many basic questions remain. One of the unsettled questions has even been whether the dimensionality of the model affects qualitative features such as the symmetry of the thermodynamic state. There is not universal agreement as to whether the low-temperature state of a finite-dimensional Ising spin glass is two-fold, as in the droplet picture of Fisher and Huse, or has a high multiplicity, as in the Parisi solution of mean field spin glass models [1].

The importance of the work by Yucesoy, Katzgraber, and Machta is that they convincingly address this long-standing question using new statistical measures. These measures can be used to examine the relationship of thermalized configurations more carefully than previous sample-averaged measures. They apply the same technique to both three-dimensional lattices (Edwards-Anderson) and mean-field (Sherrington-Kirkpatrick) graph structures. Using parallel tempering to anneal a given sample to a low temperature, they measure the probability $P(q)$ of two independent configurations having a given similarity $q$. The classic analysis has been to then average $P(0)$, the probability of observing very different configurations, over all samples. It turns out that this average measure does not distinguish the finite-dimensional and mean-field models for feasible system sizes. However, Yucesoy and collaborators find other measures of distributions to be distinct. This distinction is apparent in the probability of large peaks in the overlap distributions $P(q)$ for individual samples. The probability of peaks in the mean-field model grow with system size, while measures of the finite-dimensional model show little change with system size. Though there have been some earlier attempts to look at individual distributions in mean field models [2], this particular study has been more thorough and focused and seems to clearly distinguish the two models. Despite the fact that both mean-field and three-dimensional models are in the same difficult computational complexity class, these results indicate that dimensionality does make a difference.

Such results bring more depth to the study of complex models. The question of the thermodynamic states in spin glasses has been controversial. In part this is due to the unavoidable difficulty in simulating a problem that is NP-hard. These new results strongly suggest, however, that novel measures in small realizations can be used to distinguish qualitative behaviors. The resolution of the nature 
of states in spin glasses may clarify general ideas about complex landscapes, but regardless of that, the tools developed and the ongoing refinement of questions can serve as a guide for other studies of heterogeneous systems. These approaches include the study of the delicate effects of boundary conditions on the bulk (as has been echoed in arguments for granular materials) and the identification of rigid "backbone" structures. More sophisticated questions along with better tools will lead to a sharper understanding of the complex glassy dynamics and the relationship between computational complexity and physics. In accord with recent unsettled discussions of quantum computing [3], which often use spin glass ground state determination as a prototypical problem, this type of work also indicates that claims about qualitative behavior need to be very carefully stated and examined.

\section{References}

[1] G. Parisi, Phys. Rev. Lett. 50, 1946 (1983); D. S. Fisher and D. A. Huse, Phys. Rev. Lett. 56, 1601 (1986); C. M. Newman and D. L. Stein, Phys. Rev. Lett. 76, 515 (1996).

[2] T. Aspelmeier, A. Billoire, E. Marinari, and M. A. Moore, J. Phys. A 41, 324008 (2008).

[3] Sergio Boixo, Troels F. Rønnow, Sergei V. Isakov, Zhihui Wang, David Wecker, Daniel A. Lidar, John M. Martinis, and Matthias Troyer, arxiv:1304.4595; J. A. Smolin and G. Smith, arxiv:1305.4904. 